\title{
XLII. On some improved formulæ and methods connected with lenses
}

\section{Thomas H. Blakesley}

To cite this article: Thomas H. Blakesley (1900) XLII. On some improved formulæ and methods connected with lenses, Philosophical Magazine Series 5, 49:300, 447-453, DOI: $10.1080 / 14786440009463866$

To link to this article: http://dx.doi.org/10.1080/14786440009463866

册 Published online: 21 Apr 2009.

Submit your article to this journal

Џ Article views: 2

Q View related articles $\sqsubset$ 


\section{$\left[\begin{array}{ll}447 & ]\end{array}\right.$}

XLII. On some Improved Formula and Methods connected with Lenses. By Thomas H. Blakesley *.

TN a paper read hefore the Physical Society on June 11th, 1897,1 pointed out the connexion between the distance of an object from the first principal focus of a lens and the relation of its linear magnitude with that of the image produced : viz. that every change in that distance by the amount of the focal length of the lers produced a unit of change in the relation of these magnitudes; and I described meihods by means of which the focal length might be very accurately determined upon these grounds.

I also explained how the position of the two principal foci might be accurately determined by placing the lens upon a plane mirror and finding the spot at which if an object be placed its image inverted will coincide with it,

The rules given apply equally well to combinations of coaxial lenses as to single lenses.

Hence all focussing properties of lens-systems are really determined when the three following matters are known:-

(1) The focal length with proper sign.

(2) The position of the first principal focus.

(3) The position of the second principal focus.

The first prineipal focus is defined as the point such that if a beam of light before impact on the lens-system is diverging from it or converging to it, then after passage through the system the beam will consist of parallel rays.

The second principal focus is the point such that if before impact the beam is one of parallel rays, after passage it will consist of rays either diverging from or converging to that point.

In the present communication I wish to point out the rules by means of which, when these elements are known for any two systems, the elements for the combination of the two systems are readily obtained.

Each lens of course carries its own system of points with it, but an independent variable is necessarily introduced in combining systems at different distances.

The simplest magnitude will bo found to be the distance between the second principal focus of the first system and the first principal focus of the second system, which $\mathrm{I}$ call $k$, and which has a positive value in the direction of the propagation of light.

* Communicated by the Phyaical Society : read December 8, 1889. 
Let $u_{0}, v_{0}$ be the positions of the principal foci of the first system, and $u_{0}{ }^{\prime}, v_{0}{ }^{\prime}$ those of the second system, light being supposed to come from the right.

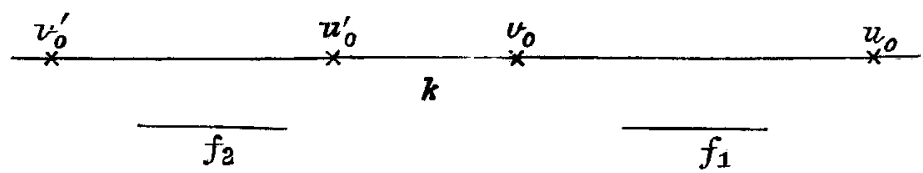

Let $f_{1}, f_{2}$ be the two focal lengths, and the length from $v_{0}$ to $u_{0}^{\prime}$ be $k$.

Then the following rules fix the elements of the whole united system.

The new first principal focus is distant from $u_{0}$ towards the right hand $\frac{f_{1}^{2}}{k}$.

The new second principal focus is distant from $v_{0}$ towards the left hand $\frac{f_{2}^{2}}{k}$.

The new focal length is $\frac{f_{1} f_{2}}{k}$.

These rules are virtually equivalent to the following statements:-

The new first principal focus is conjugate by the first system with the first principal focus of the second system.

The new second principal focus is conjugate by the second system with the second principal focus of the first system.

The points $u_{0}, v_{0}^{\prime}$ are conjugate with one another. The travel of the principal focus from $u_{0}$ to its final position being easily and accurately determinable by experiment, and having for its theoretical value $\frac{f_{1}^{2}}{k}$, it may be employed in a variety of practical ways.

The following example may illustrate how it may be used to find the focal length of a lens in the absence of a magnification apparatus.

The tubes of a telescope retaining the object-glass, but otherwise free from lenses, are placed vertically with the object-glass downwards upon a plane mirror. Crossed silk fibres are stretched across the open end, and the tubes are adjusted till the image of these fibres is in the same horizontal plane as the fibres themselves. The length of the tubes is then found to be $25.95 \mathrm{cms}$ An achromatic telescope object-glass, whose principal foci are known to be 21.12 and 19.92 from the convex and plane sides respectively, is placed upon the upper end of the telescope-tube before mentioned, the plane side being downwards. A minute triangle of white paper 
(about $3 \mathrm{sq} . \mathrm{mm}$. in area) is placed in the centre of the upper or convex side, and the tubes of the telescope, still standing on the plane mirror, are manceuvred until the image of this paper coincides with itself. The length is then found to be $25 \cdot 15 \mathrm{~cm}$. The focal length of this lens can then be determined as follows:-

The principal focus of the lower lens is above the lower surface of the upper lens by the amount of the shortening of the tubes, $i . e \cdot \overline{25 \cdot 95-25 \cdot 15}$, or 80 . But the position of the second principal focus of the upper lens is 19.92 below this face. Therefore the value of $k$ is $-(19 \cdot 92+\cdot 80)=-20 \cdot 72$, the negative value being the result of overlap in regard to the two systems.

But the amount by which the outer principal focus moves upwards is $-21 \cdot 12$.

Hence

$$
\frac{f_{1}^{2}}{-20 \cdot 72}=-21 \cdot 12 \text {. }
$$

Hence $f_{1}=-20.92$, the negative sign being of course chosen.

Again, if a convex lens, whether achromatic or not, is laid upon a plane mirror, its convex side downwards, a few drops of any refracting liquid being placed between the two, the principal focus will travel a distance depending on the index of refraction of the liquid. Care must be taken that the combination is convex in character.

If $r$ is the radius of the face common to the lens and the liquid and $\mu$ is the index of refraction of the liquid, the liquid lens will have for focal length $\frac{r}{\mu-1}$, and its first principal focus will be situated at $\frac{r}{\mu-1}$ below the plane mirror. If $v_{0}$ be taken as the distance from the wetted surface of the lens of its own second principal focus, the $k$ of this problem is clearly $\left\{\frac{r}{\mu-1}-v_{0}\right\}$.

Hence if $f$ be the focal length of the lens, and $c$ be the travel of the first principal focus due to the insertion of the liquid,

$$
\frac{f^{2}}{\frac{r}{\mu-1}-v_{0}}=c,
$$

or

$$
\frac{1}{r}\left(\frac{f^{2}}{c}+v_{0}\right)=\frac{1}{\mu-1} \text {. }
$$

Phil. Mag. S. 5. Vol. 49. No. 300. May 1900. 
If the lens constants are thoroughly known, we have an equation of the form

$$
\frac{1}{\mu-1}=\frac{\mathrm{A}}{c}+\mathrm{B}
$$

where $A$ and $B$ are both constants.

In practice it wonld be possible to determine $A$ and $B$ by observing the values of $c$ in the cases of two liquids whose indices have been determined in other ways; but it is not necessary to take this course where a single lens is employed, because in that case the radius $r$ can be expressed in terms of the focal length, the distances $u_{0}$ and $v_{0}$ of the principal foci from the anterior and posterior surfaces of the lens, and the thickness of the lens, the latter being easily measured by calipers. The formula for $\frac{1}{r}$ then is

$$
\frac{u_{0}+f}{f^{2}-u_{0} v_{0}+d f}
$$

which can be inserted in the above equation.

Or if a compound lens is employed, the value of $r$ may be determined by a spherometer; but this instrument does not furnish the most accurate means of calculating such radii. This point will receive attention below.

A telescope is an arrangement of coaxial lenses, some forming what is called the object-glass, others the eyepiece. Each of these portions may be far from simple, but each must have its own focal length and pair of principal foci. When the second principal focus of the object-glass coincides with the first principal focus of the eyepiece, the focal length of the combination is infinite, for the $k$ of this problem is equal to zero. This is often considered to be the position of adjustment for viewing distant objects ; and in fact a telescope will always have a very long focal length though the actual value will differ with different eyes. Now if a telescope is in this condition of adjustment, having an infinite focal length, we may divide the system anywhere we please, and call one part the object-glass and the other the eyepiece. All that is necessary is that the dividing surface shall be a plane at right angles to the common axis, or a sphere having its centre on the common axis. The division itself may even take place through one of the lenses.

The two portions of the system thus formed will have the following properties.

The second principal focus of one of them will always coincide with the first principal focus of the other. 
The ratio of the two focal lengths will always have the same value, the magnifying-power of the whole, wherever the division is made.

When, for the purposes of celestial photography, a telescope has a camera attached at the eye-end, the question arises how far the eyepiece must be racked out in order to obtain an image on the plate.

If the optical properties of the eyepiece are known, $i . e$. its focal length and the positions of its principal foci, then of course the distance of the camera-plate from the second principal focus is known. But the camera-plate must be in the second principal focus of the whole system. Hence the distance from the second principal focus of the eyepiece to the plate must be the travel of the principal focus due to the application of the object-glass, $i$. $e$.

$$
\frac{f^{2}}{c}=k
$$

where $f$ is the focal length of the eyepiece, $c$ is the distance of the photographic plate from the second principal focus of the eyepiece, and $k$ is the amount of racking out required from the position in which the focal length of the telescope is infinite.

If we have a lens system, and coaxial with it a mirror, either plane or spherical, and light is passed first through the lens in a direction towards the mirror, and after reflexion at the mirror, back through the lens in the reverse direction, the combination forms a virtual mirror to which the following very simple rules apply:-

The virtual mirror's surface is conjugate with the real mirror's surface by the lens.

The virtual mirror's centre of curvature is conjugate with the real mirror's centre of curvature by the lens.

These rules enable one at once to determine the general facts of the image-formation in any given case.

If the virtual mirror is a plane one, its radius of curvature is infinite, and either its surface or its centre of curvature must be at infinity, while the other must be at a finite distance.

Hence to form a virtual plane mirror either the surface or the centre of the real mirror must be in the second principal focus of the lens.

If the surface of the mirror makes this coincidence, the surface of the plane virtual mirror will be at infinity; but its centre is not so, and may be easily found experimentally, as 
the place where an object coincides with an inverted image of itself.

If the centre of curvature of the mirror coincides with the second principal focus of the lens, the centre of the curvature of the virtual plane mirror is at infinity; but the pline itself will not be so, and its position may be easily found experimentally as the place where an object coincides with an erect image of itself.

Such virtual mirrors, whether plane or not, giving erect images, are of course often formed in front of the lens; and in such cases there is not the smallest difficulty in passing an object " through the looking-glass."

The rules given above for the position of the centre of curvature and surface of the virtual mirror are equivalent to the two following mathematical formulæ:-

The surface of the virtual mirror is sitnated at a distance from the first principal focus of the lens equal to $\frac{f^{2}}{k+\frac{r}{2}}$

in a direction contrary to that in which the light is going before reflexion; and the centre of curvature of the virtual mirror is situated at a distance from the same point equal to $\frac{f^{2}}{k-\frac{r}{2}}$ measured positively in the same direction.

In these formulæ $f$ is the focal length of the lens ; $r$ is the radius of curvature of the mirror (positive if concave); $k$ is the distance from the second principal focus of the lens to the principal focus of the mirror, measured positively in the direction in which light is proceeding before reflexion.

Now suppose that the mirror coincides in curvature and position with the second face of the lens. This will be the case if either that face is silvered or placed in a pool of mercury.

The position of the centre of curvature of the virtual mirror can be found in the usual way, by coincidence with inversion of the image and object. Let it be distant $c$ from the original first principal focus of the lens, positively measured in the direction opposite to the light before reflexion.

Then by the above formula

$$
c=\frac{f^{2}}{k-\frac{r}{2}}
$$


But if $v_{0}$ be the distance of the second principal focus of the lens from the second surface, the value of $k$ will be

$$
-\left\{v_{0}+\frac{r}{2}\right\} \text {. }
$$

Hence

$$
c=-\frac{f^{2}}{v_{0}+r} \text { or } r=-\frac{f^{2}}{c}-v_{0} \text {; }
$$

all the symbols on the right hand of this last equation are known.

This affords a very convenient way of practically measuring the radius of curvature of the face of a lens, as a pool of mercury is an easy way of producing the conditions.

I have mentioned above in dealing with the index of refraction of a liquid that a knowledge of this value is important; and this optical method is in my opinion far superior to that of the spherometer in making the determination.

Those who may have followed the above remarks with care will see that I strongly advocate those optical methods of measurement which depend upon making a coincidence between the position of an object and that of its image. These coincidences may be determined with very great precision by any one accustomed to observation; and the apparatus required, though it may be made as elaborate as any others, need be no more than the simplest. A small optical bank about five inches long, a holder to slide up and down and to carry a sharply-pointed piece of white paper, four square inches of good plane mirror, a hand-lens of about 2 inches focal length to determine coincidences, and a rule and calipers to measure distances, are really all that is necessary to make determinations of an accuracy far surpassing that in vogue at present.

I have examined a lantern-projector which professed to have a focal length of 6 inches $(=15 \cdot 24 \mathrm{~cm}$.). It actually possesses one of $17.81 \mathrm{~cm}$.

A microscope objective whose nominal value was $1_{\frac{1}{2}}$ inch, made by the most eminent optician in London, was measured directly, and indirectly by taking its two component lenses separately and measuring their focal lengths and the overlap of their principal foci.

$$
\left.\begin{array}{l}
\text { The first method gave } 1.347 \\
\text { " second } 1.355
\end{array}\right\} \text { inches focal length. }
$$

The nominal value was therefore certainly in error by as much as 10 per cent. 